\title{
Tecnologías de la información y comunicación en la educación médica de posgrado
}

\author{
Information and communications technologies in postgraduate medical education
}

\author{
Carlos Alberto Orellano-Tuesta ${ }^{l}$
}

\section{Resumen}

La educación digital en programas de posgrado de medicina ofrece herramientas que se ajustan a las características del profesional actual quien busca alternativas para seguir adquiriendo conocimientos. El objetivo de este artículo es abordar las condiciones para su efectividad y señalar ciertas aplicaciones reportadas en la literatura internacional. También, se mencionan sus usos en Perú, incluyendo la experiencia en el residentado médico de la Universidad Peruana Cayetano Heredia.

Palabras clave: Tecnología de la Información, Educación de Posgrado en Medicina(DeCS).

Abstract

Digital education in postgraduate medicine programs offers tools that fit with the characteristics of the current professional who seeks alternatives to continue acquiring knowledge. The aim of this paper is to address the conditions for its effectiveness and point out certain applications reported in the international literature. Also, usages in Peru are mentioned, including the experience in the medical residency of the Peruvian University Cayetano Heredia.

Key words: Information Technology, Graduate Medical Education (MeSH).

Las tecnologías de la información y comunicación (TICs) han permitido avances en diferentes contextos, entre ellos la educación. La educación digital (o electrónica) abarca diversos tipos de intervenciones tales como las basadas en computadora en o fuera de línea, el aprendizaje digital basado en juegos, los cursos abiertos masivos en línea (o MOOCs), la simulación (realidad virtual, pacientes virtuales y entrenadores de habilidades psicomotoras) y la educación móvil; y, brinda a sus usuarios la posibilidad de adquirir conocimientos y destrezas de una manera oportuna y costo-efectiva con gran control personal $^{(1,2)}$.

La educación digital se clasifica según sus aplicaciones en: aprendizaje, enseñanza y evaluación electrónica (conocidas como e-learning, e-teaching y e-assessment, respectivamente) $)^{(3)}$. El e-learning comprende aquellos recursos a través de los cuales el estudiante accede para adquirir nuevos conocimientos por medio de actividades sincrónicas (en tiempo real) como videoconferencias y chats o asincrónicas (en tiempo diferido) tales como videoclases grabadas y foros. El e-teaching consiste en aquellas herramientas que facilitan al docente transmitir contenidos; por ejemplo, software especializado para la creación de videoclases con elementos interactivos ${ }^{(4)}$. El e-assessment se refiere al soporte de evaluaciones sumativas y formativas.
El currículo médico no es ajeno a la educación digital. Implementar esta tecnología supone evaluar qué tan efectiva y eficaz podría resultar frente a los métodos tradicionales. Para que sea exitosa: (a) hay que fomentar el uso de la tecnología en los estudiantes haciendo que sea percibida como útil y fácil de usar, aspectos que se exploran en el Modelo de Aceptación Tecnológica; y, (b) que sea interactiva, ya sea con diálogos con el docente, entre los estudiantes o con tutoriales virtuales para obtener retroalimentación sobre su compresión y desempeño, según el Marco Conversacional de Laurillard ${ }^{(5-8)}$.

\section{Efectividad de la educación digital en el posgrado}

La teoría de aprendizaje del adulto sugiere que la efectividad en intervenciones educativas difiere entre los estudiantes de pregrado y posgrado. Esto se debería a que el aprendizaje en posgrado sucede durante la práctica clínica (aprendizaje informal) y tiende a ser más autodirigido, en circunstancias donde el tiempo dedicado a los estudios está reducido por otras responsabilidades como las familiares y laborales. En consecuencia, la tecnología permitiría al estudiante organizarse mejor y cumplir con las exigencias de las asignaturas ${ }^{(9-14)}$.

\footnotetext{
${ }^{1}$ Médico psiquiatra. Magíster en informática biomédica. Docente, Departamento de Clínicas Médicas, Facultad de Medicina, Universidad Peruana Cayetano Heredia.
} 
Si bien la efectividad de la educación digital, en el marco del e-learning, se mide con la ganancia de conocimientos a través de la diferencia entre post-test y pretest, pues no sería suficiente ${ }^{(15)}$. Es necesario también analizar su diseño instruccional, el cual debería estar enmarcado dentro de alguna teoría de aprendizaje ${ }^{(2)}$. Sin embargo, aún no se cuenta con alguna herramienta validada que la evalué específicamente en cursos de posgrado ${ }^{(16)}$. Frente a este requerimiento, de Leeew y col. han reportado sus avances para el desarrollo de un cuestionario validado en concordancia con las recomendaciones de la Asociación para la Educación Médica en Europa ${ }^{(17-20)}$. Por otro lado, existen factores como el nivel de destreza para el uso de computadoras, la automotivación del estudiante y la habilidad para navegar en internet que pueden determinar el uso seguro y efectivo del elearning por lo que se sugiere revisar las competencias de los estudiantes de posgrado en cuanto a las TICs antes de su implementación ${ }^{(10)}$.

Siendo la educación digital una de las tendencias en la educación médica para el posgrado, trae consigo nuevos enfoques para el desarrollo de contenidos que incluyen al diseño instruccional basado en la reutilización de recursos para el aprendizaje y la web semántica ${ }^{(21)}$. Entiéndase como reutilización de recursos a la selección de objetos (por ejemplo, una videoclase o una lectura) de algún programa educativo previo para ser parte de los contenidos en nuevos cursos. Estos objetos se encuentran indexados en un repositorio disponible para los docentes. Esta estrategia ha sido adoptada por la International Virtual Medical School (IVIMEDS), una organización internacional de colaboración entre escuelas de medicina ${ }^{(22)}$.

En cuanto a la web semántica, esta consiste en la organización de contenidos web a través de datos que los describen (metadata). Así, estos contenidos están asociados y las búsquedas en línea se vuelven "más inteligentes". En consecuencia, a los estudiantes les facilita obtener información y compartirla con sus pares ${ }^{(23)}$.

\section{Tics aplicadas en posgrado}

Pese a su rápida evolución, hay menor número de investigaciones relacionadas a la educación digital en programas de posgrado en comparación con las desarrolladas en pregrado ${ }^{(24,25)}$. A pesar de ello, existen emprendimientos que se han reportado en la literatura y que destacan por su innovación. A continuación, se describen algunos de ellos.

\section{Aulas invertidas}

El aula invertida o flipped classroom es una forma de aprendizaje semipresencial (blend learning) en la cual se intercambian los lugares donde se llevan a cabo las actividades del aprendizaje tradicional. Es decir, los estudiantes revisan determinados contenidos antes de asistir a la clase, mientras que la clase tradicional se reemplaza por otras actividades que promueven el aprendizaje activo. Usando la tecnología, el aula invertida se divide en dos partes: actividades grupales dentro del aula y la instrucción individual basada en computadora fuera de esta, por ejemplo, a través de videoclases ${ }^{(26)}$.

Las investigaciones publicadas acerca de las aulas invertidas en el residentado médico han ido en aumento; siendo aplicadas en especialidades como emergencia, medicina interna, pediatría, neurocirugía, patología, psiquiatría, anestesiología, neurología, cirugía de tórax, radiología y ginecología y obstetricia. Las aulas invertidas poseen como componente necesario al aprendizaje activo autodirigido, que ofrece a los residentes mayor control para aprender según su propio ritmo y en momentos fuera de sus responsabilidades clínicas.

Además, este modelo es consistente con el constructivismo social, proveyéndoles de un espacio para el aprendizaje colaborativo donde demuestran la aplicación de sus conocimientos en los grupos de discusión ${ }^{(27-29)}$.

Se han reportado opiniones positivas de parte de los residentes respecto a su uso y mejoras en cuento a la obtención de conocimientos y habilidades, haciéndolas una alternativa atractiva frente al modelo tradicional ${ }^{(27)}$

\section{Revistas de Revistas y Redes Sociales}

La revista de revistas (RR) es un método que facilita la revisión entre pares y el pensamiento crítico en la educación médica basada en la evidencia ${ }^{(30)}$. Con la expansión tecnológica y formas de interacción, aparece el modelo de las RRs virtuales bajo modalidad sincrónica (por videoconferencia), asincrónica o ambas (híbrida).

Dentro de las formas asincrónicas, las redes sociales son un nuevo medio donde los profesionales se conectan con sus pares y construyen comunidades en línea con el propósito de diseminar lo último en literatura médica y trasladarlo a su práctica clínica. Su aplicación en las RRs rompe las barreras geográficas y genera inclusive debates interprofesionales. A diferencia de las sincrónicas, las RRs asincrónicas son mucho más flexibles ya que hay más tiempo disponible para que los participantes lean los artículos y envíen sus comentarios, según su propio ritmo y horarios ${ }^{(31-33)}$.

Entre las redes sociales que destacan en la literatura médica en el despliegue de RR virtuales en posgrado son los sistemas de microblogeo ${ }^{(30)}$. Hay reportes que describen su implementación en traumatología, radiología, nefrología, urología y medicina de emergencias ${ }^{(34-37)}$.

\section{MOOCs}

Los MOOCs son cursos en línea accesibles vía web de registro libre, sin límite de inscritos o requisitos previos. Contienen videos de corta duración acompañados de lecturas complementarias, cuestionarios y foros. Un meta-análisis que 
compara la eficiencia entre los MOOCs y los cursos tradicionales en educación médica no encontró diferencias significativas. Por tanto, se convierten en una alternativa viable para aquellos que desean aumentar sus conocimientos. $(38,39)$

Un gran número de MOOCs son considerados relevantes para la educación médica en posgrado. Inclusive, se ofrecen MOOCs con créditos para certificación en educación médica continua, siendo además un método económico y conveniente frente a presupuestos cada vez más cortos ${ }^{(38,40,41)}$.

\section{Gamificación}

La gamificación se define como el uso de elementos de diseño de juegos en contextos ajenos a los juegos ${ }^{(42)}$. En educación se aplica a actividades de aprendizaje para facilitar el logro de metas. Por ejemplo, la entrega de puntajes o "insignias" según el número de tareas completadas y la conformación de una tabla de clasificación (ranking) de los estudiantes. El éxito de la gamificación como intervención depende de qué tanto incrementa la motivación del estudiante. De acuerdo con la teoría de autodeterminación, esta motivación mejora cuando se cubren tres necesidades psicológicas: competencia, autonomía y relación ${ }^{(43)}$.

Recientemente, la gamificación se ha incorporado en la educación médica a fin de favorecer el compromiso, la motivación y cambios de comportamiento del estudiante ${ }^{(44)}$. Si bien no es obligatorio, la tecnología es un recurso para gamificar el aprendizaje. Respecto a posgrado, algunos reportes muestran que la gamificación, agregada a una serie de preguntas en línea como preparación, mejoró las calificaciones en residentes de medicina interna, otorrinolaringología y cirugía general $^{(45-48)}$.

\section{Evaluaciones electrónicas de competencias}

Si bien los exámenes en línea es la aplicación más importante en la evaluación electrónica parar medir conocimientos, existen otros desarrollos que buscan mejorar la recolección de calificaciones sobre competencias, específicamente en el residentado médico. Documentar este tipo de evaluaciones es una tarea difícil de emprender debido a que los docentes destinados a realizarla no poseen de tiempo disponible. El beneficio que podría recibir el residente de ser evaluado y recibir retroalimentación inmediatamente es afectado por las responsabilidades asistenciales de su docente.

Se reportó la transición de la evaluación de competencias en residentes de cirugía, comparando el uso tradicional de formularios en línea o envío de correos electrónicos y la utilización exclusiva de una novedosa plataforma a través de teléfonos inteligentes. Sus resultados mostraron que la cantidad de evaluaciones enviadas fue mayor en el grupo de que utilizó la plataforma por sus celulares, así como en el total de evaluaciones completas, siendo significativa en todos los años académicos ${ }^{(49)}$. Mientras tanto, también se ha descrito el uso de reportes en línea para proveer de retroalimentación a residentes de urología tras evaluar su desempeño en 29 procedimientos distintos; $y$, que estos reportes les permitieron documentar el progreso de sus residentes e identificar a aquellos con bajo desempeño ${ }^{(50)}$.

\section{Uso de TICs en el posgrado \\ de medicina en el Perú}

No existe en la literatura estudios cuantitativos ni cualitativos acerca de las aplicaciones de las TICs en los programas de posgrado médico de las instituciones educativas peruanas. No obstante, se hará una aproximación hacia su "estado de arte".

En el Perú, diversos programas presenciales hacen uso de aulas virtuales generalmente como repositorios de archivos, ya sean para albergar diapositivas de las clases o material de lectura para que los alumnos pueda descargarlos. Hay que destacar que algunas academias que ofrecen programas de preparación para el examen de residentado médico poseen plataformas virtuales con clases grabadas de las sesiones presenciales disponibles para aquellos suscritos bajo modalidad a distancia y también se encuentran accesibles para los matriculados en los cursos presenciales.

En cuanto a educación médica continua, el Colegio Médico del Perú ofrece a sus colegiados una plataforma virtual con cursos en línea que abordan temas relacionados atención primaria principalmente y validados para la recertificación ${ }^{(51)}$. De igual manera, la Asociación Peruana de Facultades de Medicina tiene un campus virtual con cursos dirigidos a profesionales que ejercen la docencia en salud ${ }^{(52)}$.

Cabe señalar que existen dos estudios acerca del beneficio de usar aulas virtuales. El primero trata sobre la utilización de un aula virtual para el curso de gerencia en salud bajo la modalidad de b-learning en el residentado médico de una universidad pública y señala que los médicos en formación opinaron favorablemente acerca de su implementación y que un $89 \%$ de estos recomendaría su uso para el desarrollo de otros cursos, mientras que un $91,1 \%$ consideró importante su uso como parte de su formación como médicos especialistas ${ }^{(53)}$. El segundo explora el desempeño docente en la maestría de medicina con mención para especialistas de una universidad privada y muestra como hallazgo principal que estuvo relacionado significativamente con el uso de aulas virtuales ${ }^{(54)}$. También, encontró relaciones con el uso de internet, ofimática y programas para la creación de contenidos audiovisuales.

\section{Experiencia en el Residentado Médico de la Facultad de Medicina-UPCH}

Debido a las responsabilidades asistenciales, los residentes tienen poco tiempo disponible para llevar un curso 
presencial. Los turnos regulares, las guardias programadas y las largas distancias entre las sedes hospitalarias y el campus universitario serían los principales obstáculos. Desde hace unos años, en la Facultad de Medicina de la Universidad Peruana Cayetano Heredia (UPCH) se optó por utilizar las TICs para los cursos obligatorios de Salud Mental y Metodología de la Investigación, ambos a través de la plataforma Moodle.

El curso de salud mental se lleva a distancia, con un aula virtual que contiene videoclases de corta duración presentadas por médicos psiquiatras docentes, videos animados, lecturas como material complementario y exámenes en línea antes y después de llevar la asignatura. Respecto al curso metodología de la investigación, este tiene un despliegue semipresencial; con un aula virtual donde se alojan las videoclases y los residentes "suben"" sus tareas como parte de los productos para aprobar el curso mientras que las sesiones presenciales se reservan para exponer los avances de los protocolos.

En cuanto a evaluaciones en línea, desde hace dos años, los residentes rinden el examen final de conocimientos de la especialidad días previos a la culminación de su programa de forma electrónica. Para tal fin, se acondicionan las aulas de la Facultad con laptops para cada residente desde donde acceden a nuestra plataforma en Moodle e ingresan a un aula virtual diseñada con los exámenes correspondientes. Estas evaluaciones contienen preguntas de opción múltiple de única respuesta que están configuradas para que se muestren de forma aleatorizada y de la misma manera las alternativas, y tras el envío de respuestas aparece la calificación obtenida y la revisión de preguntas.

Otra herramienta desarrollada para el año académico 2019-2020 es una plataforma web para el llenado mensual de la nueva rúbrica que evalúa los conocimientos, habilidades y actitudes del residente. Esta aplicación se desarrolló a medida usando Laravel, un framework de código abierto. Desde ahí los coordinadores de las diferentes especialidades agendan las rotaciones con sus respectivos docentes evaluadores. Es así como la plataforma envía a los correos electrónicos de estos docentes los enlaces para que completen las rúbricas de cada residente según la programación.
Referencias bibliográficas

1.Tudor Car L, Riboli-Sasco EF, Marcano Belisario JS, Nikolaou CK, Majeed A, Zary N, et al. Mobile learning for delivering health professional education. Cochrane Tobacco Addiction Group, editor. Cochrane Database of Systematic Reviews [Internet]. 15 de septiembre de 2015 [citado 13 de julio de 2019]; Disponible en: http://doi.wiley.com/10.1002/ 14651858. CD011861

2.Bajpai S, Semwal M, Bajpai R, Car J, Ho AHY. Health Professions' Digital Education: Review of Learning Theories in Randomized Controlled Trials by the Digital Health Education Collaboration. J Med Internet Res. 2019;21(3):e12912.

3.Ellaway R, Masters K. AMEE Guide 32: e-Learning in medical education Part 1: Learning, teaching and assessment. Med Teach. 2008;30(5):455-473.

4.Orellano C. Más allá del aprendizaje electrónico. Rev Medica Hered. 2018;29(2):121.

5.Wong G, Greenhalgh T, Pawson R. Internet-based medical education: a realist review of what works, for whom and in what circumstances. BMC Med Educ. 2010;10:12.

6.Katharaki M, Daskalakis S, Mantas J. Investigating the potential of e-Learning in healthcare postgraduate curricula: a structural equation model. Stud Health Technol Inform. 2010;160(Pt 1):572-575.

7.Davis FD. Perceived Usefulness, Perceived Ease of Use, and User Acceptance of Information Technology. MIS Quarterly. 1989;13(3):319.

8. Laurillard D. Rethinking university teaching: a conversational framework for the effective use of learning technologies. 2a ed. Nueva York: RoutledgeFalmer; 2002:268.

9.Knowles MS, Holton EF, Swanson RA. The adult learner: the definitive classic in adult education and human resource development. 8a ed. Abingdon: Routledge; 2015:387.

10.Kale S, Richardson B. The effective use of e-learning in postgraduate health-care students. International Journal of Therapy and Rehabilitation. 2006;13(7):299-303.
11. Coomarasamy A, Taylor R, Khan KS. A systematic review of postgraduate teaching in evidence-based medicine and critical appraisal. Med Teach. 2003;25(1):77-81.

12. Shanahan M. Being that bit older: mature students' experience of university and healthcare education. Occupational Therapy International. 2000;7(3):153-162.

13. Richardson JTE. Mature students in higher education: I. A literature survey on approaches to studying. Studies in Higher Education. 1994;19(3):309-325.

14. Henderson M, Finger G, Selwyn N. What's used and what's useful? Exploring digital technology use(s) among taught postgraduate students. Active Learning in Higher Education. 2016;17(3):235-247.

15. Taveira-Gomes $\mathbf{T}$, Ferreira $P$, Taveira-Gomes $\mathbf{I}$, Severo $M$, Ferreira MA. What Are We Looking for in Computer-Based Learning Interventions in Medical Education? A Systematic Review. J Med Internet Res. 2016;18(8):e204.

16. de Leeuw R, de Soet A, van der Horst S, Walsh K, Westerman M, Scheele F. How We Evaluate Postgraduate Medical ELearning: Systematic Review. JMIR Med Educ. 2019;5(1):e13128.

17. de Leeuw RA, Westerman M, Nelson E, Ket JCF, Scheele F. Quality specifications in postgraduate medical e-learning: an integrative literature review leading to a postgraduate medical elearning model. BMC Med Educ. 2016;16:168.

18. de Leeuw RA, Westerman M, Scheele F. Quality indicators for learner-centered postgraduate medical e-learning. Int J Med Educ. 2017;8:153-162.

19. de Leeuw RA, Walsh K, Westerman M, Scheele F. Consensus on Quality Indicators of Postgraduate Medical E-Learning: Delphi Study. JMIR Med Educ. 2018;4(1):e13.

20. Artino AR, La Rochelle JS, Dezee KJ, Gehlbach $H$. Developing questionnaires for educational research: AMEE Guide No. 87. Med Teach. 2014;36(6):463-474.

21. Harden RM. Trends and the future of postgraduate medical 
education. Emerg Med J. 2006;23(10):798-802.

22. Harden RM, Hart IR. An international virtual medical school (IVIMEDS): the future for medical education? Med Teach. 2002;24(3):261-267.

23. Abou El-Seoud S, El-Sofany H, Karam O. Semantic Web Architecture and its Impact on E-learning Systems Development. International Journal of Emerging Technologies in Learning (iJET). 2015;10(5):29.

24. Masterman E, Shuyska JA. Digitally mastered? Technology and transition in the experience of taught postgraduate students. Learning, Media and Technology. 2012;37(4):335-354.

25. Wittich CM, Agrawal A, Cook DA, Halvorsen AJ, Mandrekar JN, Chaudhry S, et al. E-learning in graduate medical education: survey of residency program directors. BMC Med Educ 2017;17(1):114.

26. Bishop J, Verleger MA. The Flipped Classroom: A Survey of the Research. En ASEE Conferences; 2013. Disponible en: https://peer.asee.org/22585

27. King AM, Gottlieb M, Mitzman J, Dulani T, Schulte SJ, Way DP. Flipping the classroom in graduate medical education: A systematic review. J Grad Med Educ. 2019;11(1):18-29.

28. King A, Boysen-Osborn M, Cooney R, Mitzman J, Misra A, Williams J, et al. Curated Collection for Educators: Five key papers about the flipped classroom methodology. Cureus. 2017;9(10):e1801.

29. Riddell J, Jhun P, Fung C-C, Comes J, Sawtelle S, Tabatabai $\mathbf{R}$, et al. Does the flipped classroom improve learning in graduate medical education? J Grad Med Educ. 2017;9(4):491496.

30. Roberts MJ, Perera M, Lawrentschuk N, Romanic D, Papa $\mathbf{N}$, Bolton D. Globalization of continuing professional development by journal clubs via microblogging: a systematic review. J Med Internet Res. 2015;17(4):e103.

31. Lewis B, Rush D. Experience of developing Twitter-based communities of practice in higher education. Research in Learning Technology. 2013;21.

32. Friedman KA, Herman SW, Fornari A. Medical education using minimal technology: achieving professional development. Med Educ Online. 2019;24(1):1622365.

33. Chetlen AL, Dell CM, Solberg AO, Otero HJ, Burton KR, Heller MT, et al. Another Time, Another Space: The Evolution of the Virtual Journal Club. Acad Radiol. 2017;24(3):273-285.

34. Palan J, Roberts V, Bloch B, Kulkarni A, Bhowal B, Dias J. The use of a virtual learning environment in promoting virtual journal clubs and case-based discussions in trauma and orthopaedic postgraduate medical education: the Leicester experience. J Bone Joint Surg Br. 2012;94(9):1170-1175.

35. Topf JM, Sparks MA, Phelan PJ, Shah N, Lerma EV, Graham-Brown MPM, et al. The evolution of the Journal Club: From Osler to Twitter. Am J Kidney Dis. 2017;69(6):82736.

36. Thangasamy IA, Leveridge M, Davies BJ, Finelli A, Stork B, Woo HH. International Urology Journal Club via Twitter: 12month experience. Eur Urol. 2014;66(1):112-127.

37. Lin M, Joshi N, Hayes BD, Chan TM. Accelerating Knowledge Translation: Reflections From the Online ALiEMAnnals Global Emergency Medicine Journal Club Experience. Ann Emerg Med. 2017;69(4):469-474.

38. Hoy MB. MOOCs 101: an introduction to massive open online courses. Med Ref Serv Q. 2014;33(1):85-91.

39. Zhao F, Fu Y, Zhang Q-J, Zhou Y, Ge P-F, Huang H-X, et al. The comparison of teaching efficiency between massive open online courses and traditional courses in medicine education: a systematic review and meta-analysis. Ann Transl Med. 2018;6(23):458.

40. Subhi Y, Andresen K, Rolskov Bojsen S, Mørkeberg Nilsson P, Konge L. Massive open online courses are relevant for postgraduate medical training. Dan Med J. 2014;61(10):A4923.

41. Liyanagunawardena TR, Williams SA. Massive open online courses on health and medicine: review. J Med Internet Res. 2014;16(8):e191.

42. Deterding S, Dixon D, Khaled R, Nacke L. From game design elements to gamefulness: defining "gamification". En: Proceedings of the 15th International Academic Mind Trek Conference on Envisioning Future Media Environments MindTrek'11. Tampere, Finlandia: ACM Press; 2011:9.

43. Deci EL, Ryan RM. Self-determination theory: A macrotheory of human motivation, development, and health. Canadian Psychology/Psychologie canadienne. 2008;49(3):182-185.

44. Rutledge C, Walsh CM, Swinger N, Auerbach M, Castro D, Dewan M, et al. Gamification in Action: Theoretical and Practical Considerations for Medical Educators. Acad Med. 2018;93(7):1014-20.

45. Nevin CR, Westfall AO, Rodriguez JM, Dempsey DM, Cherrington A, Roy B, et al. Gamification as a tool for enhancing graduate medical education. Postgrad Med J. 2014;90(1070):685-693.

46. Snyder E, Hartig JR. Gamification of board review: a residency curricular innovation. Med Educ. 2013;47(5):524-525.

47. Alexander D, Thrasher M, Hughley B, Woodworth BA, Carroll W, Willig JH, et al. Gamification as a tool for resident education in otolaryngology: A pilot study. Laryngoscope. 2019;129(2):358-361.

48. Lamb LC, DiFiori MM, Jayaraman V, Shames BD, Feeney JM. Gamified Twitter Microblogging to Support Resident Preparation for the American Board of Surgery In-Service Training Examination. J Surg Educ. 2017;74(6):986-991.

49. Green JM. An Innovative, No-cost, Evidence-Based Smartphone Platform for Resident Evaluation. J Surg Educ. 2016;73(6):e14-8.

50. Harriman D, Singla R, Nguan C. The Resident Report Card: A tool for operative feedback and evaluation of technical skills. J Surg Res. 2019;239:261-268.

51. Mendoza P. Recertificación de médicos especialistas. Academia Nacional de Medicina - Anales. 2012;33-34.

52. Campus Virtual ASPEFAM [Internet]. Lima: Asociación Peruana de Facultades de Medicina; c2017 [citado 30 de julio de 2019]. Disponible en: http://www.campus.aspefam.org.pe

53. Matzumura JP, Gutiérrez H. Utilización de tecnología información, comunicación y aula virtual en la enseñanza de la asignatura de gerencia en salud para los médicos residentes de la Facultad de Medicina de la Universidad Nacional Mayor de San Marcos, 2015. Anales de la Facultad de Medicina. 2016;77(3):251.

54. Burneo Cortes CG. Uso de aulas virtuales y desempeño docente en maestrías de la Facultad de Medicina Humana de la Universidad de San Martín de Porres 2018 [Tesis de Maestría]. [Lima, Perú]: Universidad de San Martín de Porres; 2019.

Citar como: Orellano-Tuesta CA. Tecnologías de la información y comunicación en la educación médica de posgrado. Diagnóstico (Lima). 2019;58(3):143-147. DOI: 10.33734/diagnostico.v58i3.63

Correspondencia: Carlos Alberto Orellano-Tuesta Correo electrónico: carlos.orellano@upch.pe 